\title{
Une inégalité maximale sous-gaussienne sur les espaces de tentes
}

\author{
par \\ E. LABEYE-Voisin (Lyon)
}

\begin{abstract}
We introduce a maximal function (denoted by $\bar{\pi}$ ) on the tent spaces $T^{p}\left(\mathbb{R}_{+}^{n+1}\right), 0<p<\infty$, of Coifman, Meyer and Stein [8]. We prove a good- $\lambda$ estimate of subgaussian type for this maximal function and for the square function of tent spaces, leading to integrability results for $\bar{\pi}$. We deduce convergence results for the singular integral defining $\pi$.
\end{abstract}

1. Introduction. On fixe deux entiers strictement positifs $n$ et $m$. On se place sur le demi-espace euclidien $\mathbb{R}_{+}^{n+1}=\mathbb{R}^{n} \times \mathbb{R}_{+}^{*}$. Par la suite $\theta \in \mathbb{R}^{n}$ désignera un point du bord et $(x, y),(s, t) \in \mathbb{R}^{n} \times \mathbb{R}_{+}^{*}$ des points du demiespace. Soit $\phi \in \mathbb{C}^{\infty}\left(\mathbb{R}^{n}, \mathbb{R}^{m}\right)$ à support dans la boule unité et supposée fixée dans la suite. On note $\phi_{y}(\theta-x)=y^{-n} \phi\left(\frac{\theta-x}{y}\right)$ le noyau associé.

Pour $0<p \leq 1$, on notera $\left(\mathcal{H}_{p}\right)$ la condition d'annulation supplémentaire suivante :

$$
\begin{aligned}
\left(\mathcal{H}_{p}\right) \quad \int x^{\gamma} \phi(x)=0 \quad & \text { pour tout multi-indice } \gamma=\left(\gamma_{1}, \ldots, \gamma_{n}\right) \in \mathbb{N}^{n} \\
& \text { tel que }|\gamma|=\gamma_{1}+\ldots+\gamma_{n} \leq\lfloor n / p-n\rfloor,
\end{aligned}
$$

où $\lfloor x\rfloor$ désigne la partie entière de $x$. Dans toute la suite la condition $\left(\mathcal{H}_{1}\right)$ sera toujours supposée vérifiée.

À toute application $F: \mathbb{R}_{+}^{n+1} \rightarrow \mathbb{R}^{m}$ localement de carré intégrable et à tout $\alpha>0$ on associe la fonction quadratique

$$
A_{\alpha}(F, \theta)=\left(\int_{\Gamma_{\alpha}(\theta)} d s d t t^{1-n}|F(s, t)|^{2}\right)^{1 / 2}
$$

où $\Gamma_{\alpha}(\theta)=\left\{(x, y) \in \mathbb{R}_{+}^{n+1} ;|x-\theta|<\alpha y\right\}$ et $|F(s, t)|$ désigne la norme euclidienne de $F(s, t)$ dans $\mathbb{R}^{m}$. On convient de plus que par défaut, l'ouverture d'un cône vaut 1 (i.e. $A(F)=A_{1}(F)$ ).

2000 Mathematics Subject Classification: 42B25, 42B30, 31B25.

Key words and phrases: tent spaces, maximal inequality, subgaussian estimate. 
Rappelons la définition des espaces de tentes introduits par CoifmanMeyer-Stein [8] et notés $T^{p}$ ("tent spaces") :

$$
T^{p}=\left\{F: \mathbb{R}_{+}^{n+1} \rightarrow \mathbb{R}^{m} ; A(F) \in L^{p}\left(\mathbb{R}^{n}\right)\right\}, \quad 0<p<\infty,
$$

on les munit de la "norme" $\|F\|_{T^{p}}=\|A(F)\|_{p}$. Ces espaces sont apparentés aux espaces de Hardy $H^{p}$ habituels. Sur ces espaces Coifman, Meyer et Stein introduisent l'opérateur $\pi$ défini a priori sur l'espace $T_{c}$ des fonctions tentes $F$ à support compact dans $\mathbb{R}_{+}^{n+1}$ par

$$
\pi(F, \theta)=\int_{\Gamma(\theta)} d s d t \phi_{t}(\theta-s) \cdot F(s, t)
$$

pour lequel ils prouvent le :

ThÉorème 1.1 (cf. [8] et [18, chap. IV.6]). Sous la condition d'annulation $\left(\mathcal{H}_{1}\right)$, pour tout $n /(n+1)<p<\infty$, l'opérateur $\pi$ s'étend en un opérateur continu de $T^{p}$ sur $H^{p}$.

Pour $0<p \leq n /(n+1)$, si $\phi$ vérifie de plus la condition d'annulation $\left(\mathcal{H}_{p}\right)$ alors l'opérateur $\pi$ s'étend encore en un opérateur continu de $T^{p}$ sur $H^{p}$.

Cet opérateur peut remplacer (quoiqu'imparfaitement), dans le contexte de l'analyse réelle, l'intégrale stochastique des probabilistes. De tels opérateurs ont déjà été étudiés et on peut remarquer avec Gundy [10] (voir aussi [18, chap. IV.6]) que si $F(s, t)=\nabla u(s, t)$ avec $u$ extension harmonique à $\mathbb{R}_{+}^{n+1}$ de $f \in L^{2}\left(\mathbb{R}^{n}\right)$, alors $\pi$ vu comme opérateur agissant sur $f$ redonne l'opérateur identité sur $L^{2}\left(\mathbb{R}^{n}\right)$ et les transformées de Riesz si on fait le bon choix de noyau $\phi$ (cf. [10, pp. 18 à 30]).

L'existence de cette extension n'assure pas a priori que l'on puisse écrire et manipuler sereinement une formule comme $\int \phi_{y}(\theta-x) \cdot F(x, y) d x d y$ pour tout $F \in T^{p}$ et $\theta \in \mathbb{R}^{n}$. En effet l'intégrande n'est pas en général absolument intégrable et le procédé utilisé pour construire cette intégrale ne lui donne un sens qu'en tant que classe de fonctions dans les espaces $H^{p}$, $0<p<\infty$, et donc pas en tout point $\theta$ de $\mathbb{R}^{n}$ (en presque tout point seulement). Dès lors, toute notion faisant intervenir les valeurs de $\pi$ pour une famille non dénombrable de fonctions tentes ne peut avoir de sens (ponctuel, presque partout ou dans les espaces $H^{p}$ ). Par exemple, si $\mathcal{L}$ est un ensemble non dénombrable de parties de $\mathbb{R}_{+}^{n+1}$, et $F \in \bigcup_{1<p<\infty} T^{p}$, l'application $\theta \mapsto \sup _{V \in \mathcal{L}} \pi\left(F \mathbb{1}_{V}, \theta\right)$ est en général mal définie. Pourtant, comme nous le verrons, il est possible de donner presque partout une définition "ponctuelle" à la troncature de l'opérateur $\pi$ par une famille raisonnable de parties de $\mathbb{R}_{+}^{n+1}$. C'est là un des principaux buts de ce papier. Cela permet de donner à cet opérateur une partie de l'intérêt technique que présentent les intégrales stochastiques en probabilités. En effet nous obtenons ainsi un contrôle du 
comportement de $\pi$ sous l'effet d'une troncature par des "saw-tooth regions", lesquelles jouent souvent en analyse réelle le rôle des temps d'arrêts en probabilités. C'est ce qui motive l'introduction de notre fonction maximale dans la section suivante.

Cela nous a permis, par exemple dans [12] et [13], d'étudier en norme la dépendance de la fonctionnelle densité de l'intégrale d'aire $D^{a} u$ par rapport à la fonction harmonique $u$, et d'obtenir des résultats analogues à ceux obtenus en probabilités par Barlow et Yor (cf. [5]) sur le temps local de martingales continues. Dans cet exemple, les espaces de tentes interviennent de façon essentielle au travers d'une formule due dans ce contexte à Gundy, Bañuelos et Moore (cf. [11] et [4]) et très proche de la formule "à la Tanaka" de Chevalier (cf. [7]) qui permet de décomposer la densité d'intégrale d'aire sous la forme : $D^{a} u=(u-a)^{+}+\pi\left(\nabla(u-a)^{+}\right)$. Le noyau $\phi$ intervenant dans cette décomposition constitue un exemple pratique de noyau pour lequel seule la condition d'annulation $\left(\mathcal{H}_{1}\right)$ est vérifiée.

2. Présentation des résultats. Dans toute cette section on suppose seulement que $\phi$ vérifie l'hypothèse d'annulation $\left(\mathcal{H}_{1}\right)$.

Notons $\mathcal{L} \subset \mathbb{C}^{0}\left(\mathbb{R}^{n}\right)$ l'ensemble des fonctions positives $w$, lipschitziennes sur $\mathbb{R}^{n}$ (c'est-à-dire telles que $|w(x)-w(y)| \leq|x-y|$ pour tout $x, y \in \mathbb{R}^{n}$ ). On associera à tout élément $w \in \mathcal{L}$ son épigraphe $W=\left\{(x, y) \in \mathbb{R}^{n} \times\right.$ $\left.\mathbb{R}^{+} ; y>w(x)\right\}$, en convenant de les noter de la même lettre (minuscule pour la fonction, majuscule pour l'épigraphe associé). Notons aussi $\mathcal{L}^{*}$ le sous-ensemble de $\mathcal{L}$ constitué des fonctions $w$ strictement positives.

Pour tout intervalle $I$ de $\mathbb{R}_{+}$on note de plus $\pi^{I}(F)$ (resp. $\left.A^{I}(F), W^{I}\right)$ l'ensemble $\pi\left(F \mathbb{1}_{\mathbb{R}^{n} \times I}\right)$ (resp. $\left.A\left(F \mathbb{1}_{\mathbb{R}^{n} \times I}\right), W \cap\left(\mathbb{R}^{n} \times I\right)\right)$.

Commençons par quelques résultats de régularité sur $\pi$ qui vont nous permettre de donner une définition ponctuelle de notre fonction maximale.

Pour $D \subset \mathbb{R}^{n+1}$, nous noterons $y_{D}=\inf \left\{y>0 ; \exists x \in \mathbb{R}^{n}:(x, y) \in D\right\}$ et aussi $y_{D}(\theta)=y_{D \cap \Gamma(\theta)}$.

LEMme 2.1. (a) Il existe une constante $c_{1}$ ne dépendant que de $\phi$ et $n$ telle que pour tout borélien $D \subset \Gamma(\theta)$ et tout épigraphe $V$ de fonction lipschitzienne, on ait la majoration

$$
\left|\pi^{] y_{V}(\theta),+\infty[}\left(F \mathbb{1}_{D \backslash V}, \theta\right)\right| \leq c_{1} A^{] y_{V}(\theta),+\infty[}\left(F \mathbb{1}_{D \backslash V}, \theta\right) .
$$

(b) Il existe une constante $c_{2}$ ne dépendant que de $\phi$ et $n$ telle que, pour tout $\theta \in \mathbb{R}^{n}$, tout borélien $D$, tout $x \in \mathbb{R}^{n}$ et toute fonction tente $F$, on ait

$$
\left|\pi\left(F \mathbb{1}_{D}, x\right)-\pi\left(F \mathbb{1}_{D}, \theta\right)\right| \leq c_{2} \frac{|\theta-x|}{y_{D}}\left(A\left(F \mathbb{1}_{D}, \theta\right)+A\left(F \mathbb{1}_{D}, x\right)\right) .
$$


Démonstration. Commençons par établir le (a). D'après l'inégalité de Cauchy-Schwarz

$$
\begin{aligned}
& \int_{\mathbb{R}_{+}^{n+1}}\left|\phi_{y}(\theta-x)\right||F(x, y)| \mathbb{1}_{D \backslash V}(x, y) d x d y \\
& \leq\|\phi\|_{\infty} A\left(F \mathbb{1}_{D \backslash V}, \theta\right)\left(\int_{\Gamma(\theta)} \frac{d x d y}{y^{n+1}} \mathbb{1}_{D \backslash V}(x, y)\right)^{1 / 2} .
\end{aligned}
$$

Il nous reste à estimer (en fonction de $y$ ) la mesure des tranches $E_{y}=$ $\left\{x \in \mathbb{R}^{n} ;(x, y) \in \Gamma(\theta) \cap D \backslash V\right\}:$

$$
\begin{aligned}
\left|E_{y}\right| & \leq|B(\theta, y)|-\left|\left\{x \in \mathbb{R}^{n} ;(x, y) \in V\right\}\right| \\
& \leq c_{n}\left(y^{n}-\left(y-y_{V}(\theta)\right)^{n}\right) \leq n c_{n} y^{n-1} y_{V}(\theta) .
\end{aligned}
$$

En effet, il y a au moins une boule de rayon $y-y_{V}(\theta)$ dans chaque tranche $\left\{x \in \mathbb{R}^{n} ;(x, y) \in V\right\}$ de $V ;$ ce qui donne le résultat.

Passons à la preuve du (b). Elle est dans l'esprit du lemme 6 de [3], et s'obtient par les mêmes techniques :

$$
\begin{aligned}
\left|\pi\left(F \mathbb{1}_{D}, x\right)-\pi\left(F \mathbb{1}_{D}, \theta\right)\right| & \\
\leq & \left(\int_{D \cap(\Gamma(\theta) \cup \Gamma(x))} t^{n-1}\left|\phi_{t}(\theta-s)-\phi_{t}(x-s)\right|^{2} d s d t\right)^{1 / 2} \\
& \times\left(\int_{D \cap(\Gamma(\theta) \cup \Gamma(x))} \frac{d s d t}{t^{n-1}}|F(s, t)|^{2}\right)^{1 / 2} \\
\leq & \|D \phi\|_{\infty}|\theta-x|\left(\int_{D} \frac{d s d t}{t^{n+3}}\right)^{1 / 2}\left(\int_{D \cap(\Gamma(\theta) \cup \Gamma(x))} \frac{d s d t}{t^{n-1}}|F(s, t)|^{2}\right)^{1 / 2} \\
\leq & \sqrt{c_{n}}\|D \phi\|_{\infty} \frac{|\theta-x|}{y_{D}}\left(\int_{D \cap(\Gamma(\theta) \cup \Gamma(x))} \frac{d s d t}{t^{n-1}}|F(s, t)|^{2}\right)^{1 / 2} .
\end{aligned}
$$

Les résultats suivants permettent d'estimer la régularité de la partie "haute" de $\pi(F)$.

Lemme 2.2. Pour tout borélien $W \subset \mathbb{R}_{+}^{n+1}$, tout $0<p \leq 1$ et tout $\alpha>-n / p$, il existe une constante $c>0$ ne dépendant que de $n, \alpha, p$ et $\phi$ telle que, pour toute fonction tente $F$,

$$
\int_{W} \frac{|F(x, y)|}{y^{n+\alpha}} d x d y \leq c\left(\int_{\mathbb{R}^{n}} d \theta A\left(F \mathbb{1}_{W}, \theta\right)^{p} y_{W}(\theta)^{-n-\alpha p}\right)^{1 / p} .
$$

Corollaire 2.3. Soient $W \in \mathcal{L}, F \in T^{p}, 0<p<\infty$ et

$$
E=\left\{\theta \in \mathbb{R}^{n} ; w(\theta)>0\right\} .
$$


(i) L'intégrale $\int_{W} \phi_{y}(\theta-x) \cdot F(x, y) d x d y$ est absolument convergente en tout point $\theta$ de $E$ et il existe une constante $c_{3}$ ne dépendant que de $n, \phi$ et $p$ telle que

$$
\int_{W}\left|\phi_{y}(\theta-x)\right||F(x, y)| d x d y \leq c_{3} y_{W}(\theta)^{-n / p}\left\|F \mathbb{1}_{W}\right\|_{T^{p}} .
$$

(ii) L'application $\theta \mapsto \int_{W} \phi_{y}(\theta-x) \cdot F(x, y) d x d y$ a les mêmes propriétés de régularité (continuité et différentiabilité) que $\phi$ en tout point de $E$.

Démonstration du lemme 2.2. Le cas $p=1$ se déduit directement de l'inégalité de dualité

$$
\int_{\mathbb{R}_{+}^{n+1}} F(x, y) G(x, y) y d x d y \leq c_{n} \int_{\mathbb{R}^{n}} A(F, \theta) A(G, \theta) d \theta
$$

du théorème 2 de $[8]$ appliqué à $G(x, y)=y^{-(n+\alpha+1)} \mathbb{1}_{W}(x, y)$.

Le cas général $0<p \leq 1$ nécessite d'autres arguments. Soit $\mathcal{F}$ la famille des cubes dyadiques de $\mathbb{R}^{n}$. À chaque cube $Q \in \mathcal{F}$ on associe le pavé de $\mathbb{R}_{+}^{n+1}$ :

$$
T_{Q}=Q \times[\sqrt{n} \ell(Q), 2 \sqrt{n} \ell(Q)[
$$

où $\ell(Q)$ désigne la longueur des côtés de $Q$. En utilisant la sous-additivité de $x \mapsto x^{p}$ pour $p \leq 1$, puis l'inégalité de Cauchy-Schwarz on obtient

$$
\begin{aligned}
& \left(\int_{\mathbb{R}_{+}^{n+1}} \frac{|F(x, y)|}{y^{n+\alpha}} \mathbb{1}_{W}(x, y) d x d y\right)^{p} \\
& \quad \leq \sum_{Q \in \mathcal{F}}\left(\int_{T_{Q}} \frac{|F(x, y)|^{2}}{y^{n-1}} \mathbb{1}_{W}(x, y) d x d y\right)^{p / 2}\left(\int_{T_{Q}} \frac{1}{y^{n+1+2 \alpha}} d x d y\right)^{p / 2} .
\end{aligned}
$$

Par construction la première intégrale du membre de droite est égale à $A\left(F \mathbb{1}_{T_{Q} \cap W}, \theta\right)$ pour tout $\theta \in Q$, d'où

$$
\begin{gathered}
\left(\int_{\mathbb{R}_{+}^{n+1}} \frac{|F(x, y)|}{y^{n+\alpha}} \mathbb{1}_{W}(x, y) d x d y\right)^{p} \leq c \sum_{Q \in \mathcal{F}} \frac{\ell(Q)^{-\alpha p}}{|Q|} \int_{Q} d \theta A\left(F \mathbb{1}_{T_{Q} \cap W}, \theta\right)^{p} \\
\leq c \int_{\mathbb{R}^{n}} d \theta A\left(F \mathbb{1}_{W}, \theta\right)^{p} \sum_{\substack{Q \in \mathcal{F} \\
T_{Q} \cap W \neq \emptyset}} \ell(Q)^{-n-\alpha p} \mathbb{1}_{Q}(\theta) .
\end{gathered}
$$

Or pour $\theta \in Q$ et $T_{Q} \cap W \neq \emptyset$ on a $\ell(Q) \geq(2 \sqrt{n})^{-1} y_{W}(\theta)$. Ainsi la série du membre de droite est une série géométrique de raison $2^{-n-\alpha p}<1$ et de premier terme $c_{n} y_{W}(\theta)^{-n-\alpha p}$. Le résultat s'en déduit directement.

Le corollaire découle directement de ces estimations par les théorèmes classiques de dérivation sous le signe intégrale.

Nous pouvons dès lors introduire notre fonction maximale : 
DÉfinition 2.4. Pour toute fonction tente $F \in T^{p}, 0<p<\infty$, et tout $\theta \in \mathbb{R}^{n}$, on pose

$$
\bar{\pi}(F, \theta)=\sup _{W \in \mathcal{L}^{*}}\left|\pi\left(F \mathbb{1}_{W}, \theta\right)\right| .
$$

D'après ce qui précède, ce supremum non dénombrable a bien un sens en tout $\theta \in \mathbb{R}^{n}$ puisque pour chaque $W \in \mathcal{L}^{*}, F \mathbb{1}_{W}$ vérifie bien les hypothèses de support du corollaire 2.3 ci-dessus. $\pi\left(F \mathbb{1}_{W}, \theta\right)$ est donc bien définie en tout $\theta \in \mathbb{R}^{n}$ et continue. Ceci fait de $\bar{\pi}(F)$ une fonction semicontinue inférieurement sur $\mathbb{R}^{n}$.

Dans notre étude de cette fonction maximale, notre principal outil sera un résultat d'intégrabilité exponentielle de la "partie basse" de $\pi$ dû à Chang, Wilson, Wolff [6] et Bañuelos, Klemes, Moore (cf. [1], [2], [15]).

Proposition 2.5 (cf. [15] par exemple). Soit $\alpha>32 \sqrt{n}$. Sous l'hypothèse d'annulation $\left(\mathcal{H}_{1}\right)$, pour tout $\beta>0$, il existe deux constantes $c_{4}$ et $c_{5}$ ne dépendant que de $n, \phi, \alpha$ et $\beta$ telles que, pour tout cube $Q$ de $\mathbb{R}^{n}$ et toute fonction tente $F \in \bigcup_{1<p<\infty} T^{p}$, on ait

$$
\frac{1}{|Q|} \int_{Q} d \theta \exp \left(\pi^{] 0, \beta \ell(Q)]}(F, \theta)-c_{4} A_{\alpha}^{] 0, \beta \ell(Q)]}(F, \theta)^{2}\right) \leq c_{5}
$$

où $\ell(Q)$ désigne la longueur des côtés du cube $Q$.

Nous allons voir comme corollaire des théorèmes suivants que cette inégalité est encore vraie si on remplace l'opérateur $\pi$ par l'opérateur maximal $\bar{\pi}$. Notre principal résultat est l'estimation "locale" suivante :

ThÉorÈme 2.6. Soit $\alpha>32 \sqrt{n}$. Si $\phi$ vérifie la condition d'annulation $\left(\mathcal{H}_{1}\right)$, alors pour tout réel $\beta>0$, il existe deux constantes positives $c_{6}$ et $c_{7}$ ne dépendant que de $n, \alpha, \beta$ et $\phi$ telles que, pour tout cube $Q$ de $\mathbb{R}^{n}$ et toute fonction tente $F$, on ait

$$
\left|\left\{x \in Q ; \bar{\pi}^{0, \beta \ell(Q)[}(F, x)>\lambda\right\}\right| \leq c_{7} \exp \left(-c_{6} \frac{\lambda^{2}}{\left\|A_{\alpha}^{] 0, \beta \ell(Q)[}(F)\right\|_{\infty}^{2}}\right)|Q| .
$$

De cette majoration de la fonction de répartition de la "partie basse" de $\bar{\pi}(F)$ on déduira les inégalités suivantes :

ThÉorÈme 2.7. Soit $\alpha>96 \sqrt{n}$. Si $\phi$ vérifie la condition d'annulation $\left(\mathcal{H}_{1}\right)$, alors il existe deux constantes positives $c_{8}$ et $c_{9}$ ne dépendant que de $n, \alpha$ et $\phi$ telles que, pour tous $\lambda>0, \delta>0$ et $\gamma>3$, et toute fonction tente $F$, on ait

$$
\begin{aligned}
\mid\left\{\theta \in \mathbb{R}^{n} ; \bar{\pi}(F, \theta)>\gamma \lambda\right. & \text { et } \left.A_{\alpha}(F, \theta) \leq \delta \lambda\right\} \mid \\
\leq & c_{9} \exp \left(-c_{8}(\gamma / \delta)^{2}\right)\left|\left\{\theta \in \mathbb{R}^{n} ; \bar{\pi}(F, \theta)>\lambda\right\}\right| .
\end{aligned}
$$

Corollaire 2.8. Sous les mêmes hypothèses que celles du théorème 2.7, pour toute fonction tente $F \in \bigcup_{0<p<\infty} T^{p}$ : 
(a) Pour tout $0<p<\infty$, il existe une constante $c_{p}>0$ ne dépendant que de $n$, $\phi$ et $p$ telle que $\|\bar{\pi}(F)\|_{p} \leq c_{p}\|F\|_{T^{p}}$. On a de plus $c_{p}=O(\sqrt{p})$ quand $p$ tend vers $+\infty$.

(b) Pour tout $0<p<\infty$, il existe deux constantes $c_{10}$ et $c_{p}^{\prime}\left(c_{10}\right.$ ne dépendant que de $n, \alpha$ et $\phi ; c_{p}^{\prime}$ dépendant en plus de $\left.p\right)$ telles que

$$
\int_{\mathbb{R}^{n}} d \theta\left(\exp \left(c_{10} \frac{\bar{\pi}(F, \theta)^{2}}{A_{\alpha}(F, \theta)^{2}}\right)(\bar{\pi}(F, \theta))^{p}\right) \leq c_{p}^{\prime}\|F\|_{T^{p}}^{p} .
$$

(c) Pour tout $\beta>0$, il existe deux constantes $c_{11}$ et $c_{12}$ ne dépendant que de $n, \phi, \alpha$ et $\beta$ telles que, pour tout cube $Q$ de $\mathbb{R}^{n}$ et toute fonction tente $F \in \bigcup_{0<p<\infty} T^{p}$, on ait

$$
\frac{1}{|Q|} \int_{Q} d \theta \exp \left(\bar{\pi}^{0, \beta \ell(Q)]}(F, \theta)-c_{11} A_{\alpha}^{0, \beta \ell(Q)]}(F, \theta)^{2}\right) \leq c_{12}
$$

où $\ell(Q)$ désigne la longueur des côtés du cube $Q$.

Démonstration du théorème 2.6. On note $I=] 0, \beta \ell(Q)]$. Quitte à multiplier $F$ par un scalaire bien choisi, on peut supposer $\left\|A^{I}(F)\right\|_{\infty}=1$. On peut de plus se contenter de ne considérer que les $\lambda>K$ avec $K=2\left(c_{1}+c_{2}\right)$ (cf. lemme 2.1), une constante ne dépendant que de $\phi$ et $n$.

Notons $E$ l'ensemble $\left\{\theta \in Q ; \bar{\pi}^{I}(F, \theta)>\lambda\right\}$. Grâce au lemme suivant, pour chaque $\theta \in E$, on construit dans $\Gamma^{I}(\theta)$ un domaine $V_{\theta}$ correspondant à un (il n'y a pas unicité) "premier instant" d'atteinte de la valeur $\lambda$ par

$$
V \mapsto\left|\pi^{I}\left(F \mathbb{1}_{V}, \theta\right)\right|
$$

Lemme 2.9. Soient $\left.\theta \in \mathbb{R}^{n}, I \subset\right] a, b\left[\subset \mathbb{R}_{+}^{*}\right.$, et $F \in T^{p}, 0<p<\infty$. Pour tout $0<\lambda<\bar{\pi}^{I}(F, \theta)$, il existe un domaine $V \in \mathcal{L}^{*}$ tel que $V \subset$ $\Gamma^{] a,+\infty[}(\theta)$ et

$$
\left|\pi^{I}\left(F \mathbb{1}_{V}, \theta\right)\right|=\bar{\pi}^{I}\left(F \mathbb{1}_{V}, \theta\right)=\lambda .
$$

Un tel domaine sera noté $V_{\theta}$.

Démonstration du lemme. Notons $\varepsilon=\bar{\pi}^{I}(F, \theta)-\lambda>0$. On va construire, par récurrence, une suite $\left(V_{n}, n \in \mathbb{N}^{*}\right)$ décroissante de parties (i.e. $V_{n} \subseteq V_{m}$ si $n>m$ ) tels que pour tout $n \in \mathbb{N}^{*}$ :

$$
\left\{\begin{array}{l}
V_{n} \in \mathcal{L}^{*} \quad \text { et } \quad V_{n} \subset \Gamma^{] a,+\infty[}(\theta), \\
\left|\pi^{I}\left(F \mathbb{1}_{V_{n}}, \theta\right)\right|=\lambda \text { et } \quad \bar{\pi}^{I}\left(F \mathbb{1}_{V_{n}}, \theta\right) \leq \lambda+\varepsilon / n
\end{array}\right.
$$

Supposons ces $V_{n}$ construits, posons $V=\bigcap_{n \in \mathbb{N}^{*}} V_{n}$ et montrons que ce $V$ convient.

Tout d'abord $V \neq \emptyset$. En effet, d'après le (i) du corollaire 2.3 on voit aisément que $\left\{y_{W} ;\left|\pi^{I}\left(F \mathbb{1}_{W}, \theta\right)\right|=\lambda\right\}$ est majoré par $y_{\max }=\left(c_{3}\|F\|_{T^{p}} / \lambda\right)^{p / n}$, donc $\Gamma^{2} y_{\max },+\infty\left[(\theta) \subset W\right.$ pour tout $W$ tel que $\left|\pi^{I}\left(F \mathbb{1}_{W}, \theta\right)\right|=\lambda$. Notons 
que $V_{n} \rightarrow V$ dans $\mathcal{L}$ lorsque $n \rightarrow \infty$ dans $\mathcal{L}$ et du fait de la croissance de $\bar{\pi}$ par rapport aux domaines, on en déduit que

$$
\left|\pi^{I}\left(F \mathbb{1}_{V}, \theta\right)\right|=\bar{\pi}^{I}\left(F \mathbb{1}_{V}, \theta\right)=\lambda .
$$

Construisons donc notre suite $V_{n}, n \in \mathbb{N}^{*}:$

Prenons $V_{1} \in \mathcal{L}^{*}$ tel que $V_{1} \subset \Gamma^{a,+\infty[}(\theta)$ et $\left|\pi^{I}\left(F \mathbb{1}_{V_{1}}, \theta\right)\right|=\lambda$ (son existence est assurée par le fait que $\bar{\pi}^{I}(F, \theta)>\lambda$ et $\bar{\pi}^{I}\left(F \mathbb{1}_{\Gamma]^{2 y \max },+\infty[(\theta)}, \theta\right)$ $<\lambda)$. On a alors $\bar{\pi}^{I}\left(F \mathbb{1}_{V_{1}}, \theta\right) \leq \bar{\pi}^{I}(F, \theta)=\lambda+\varepsilon$. Donc $V_{1}$ vérifie bien $\left(H_{1}\right)$.

Supposons $V_{1}, \ldots, V_{n}$ trouvés tels que $V_{i}$ vérifie $\left(H_{i}\right)$ pour $1 \leq i \leq n$. Comme $\bar{\pi}^{I}\left(F \mathbb{1}_{V_{n}}, \theta\right) \in[\lambda, \lambda+\varepsilon / n]$, on peut trouver $W_{n} \subseteq V_{n}$ tel que

$$
\bar{\pi}^{I}\left(F \mathbb{1}_{W_{n}}, \theta\right) \in[\lambda, \lambda+\varepsilon /(n+1)] \quad \text { puisque } \Gamma\left(\theta, 2 y_{\max }\right) \subset V_{n} .
$$

On prend alors $V_{n+1} \subseteq W_{n}$ tel que $\left|\pi^{I}\left(F \mathbb{1}_{V_{n+1}}, \theta\right)\right|=\lambda$. Et bien évidemment

$$
\bar{\pi}^{I}\left(F \mathbb{1}_{V_{n+1}}, \theta\right) \leq \bar{\pi}^{I}\left(F \mathbb{1}_{W}, \theta\right) \leq \lambda+\varepsilon /(n+1) .
$$

L'ensemble $V_{n+1}$ ainsi trouvé vérifie bien $\left(H_{n+1}\right)$.

Nous obtenons ainsi notre suite et la démonstration du lemme est terminée.

Notons $Q_{\theta}=Q\left(\theta, y_{V_{\theta}}\right)$ et $Q_{\theta}^{\prime}=Q\left(\theta, 11 y_{V_{\theta}}\right)$ les cubes de $\mathbb{R}^{n}$ de centre $\theta$ et diamètres respectivement $y_{V_{\theta}}$ et $11 y_{V_{\theta}}$. Par un lemme de recouvrement de type Vitali (cf. [17, p. 9] par exemple) on extrait un sous-ensemble fini $\Delta$ de $E$ tel que :

(i) les $\left\{Q_{\theta}^{\prime} ; \theta \in \Delta\right\}$ soient deux à deux essentiellement disjoints;

(ii) $|E| \leq c \sum_{\theta \in \Delta}\left|Q_{\theta}\right|$.

On pose alors $V=\bigcup_{\theta \in \Delta} V_{\theta}$. On exploite les propriétés de régularité locales de $\pi$ (lemme 2.1) pour voir que pour tout $x \in \bigcup_{\theta \in \Delta} Q_{\theta}$ on a $\left|\pi^{I}\left(F \mathbb{1}_{V}, x\right)\right| \geq$ $\lambda / 2$ et $A_{\alpha}^{I}\left(F \mathbb{1}_{V}, x\right) \leq 1$ : remarquons en effet que pour tout $\theta \in \Delta$ et tout $x \in Q_{\theta}$ on a $y_{V \backslash V_{\theta}}(x) \geq y_{V_{\theta}}(x)$. On peut donc estimer

$$
\begin{aligned}
\mid \pi^{I}\left(F \mathbb{1}_{V},\right. & x)-\lambda \mid \\
& \leq\left|\pi^{I}\left(F \mathbb{1}_{V}, x\right)-\pi^{I}\left(F \mathbb{1}_{V_{\theta}}, x\right)\right|+\left|\pi^{I}\left(F \mathbb{1}_{V_{\theta}}, x\right)-\pi^{I}\left(F \mathbb{1}_{V_{\theta}}, \theta\right)\right| \\
& \leq\left(c_{1}+c_{2} \frac{|\theta-x|}{y_{V_{\theta}}}\right) A^{] y_{V_{\theta}},+\infty[\cap I}(F, \theta) \leq c_{1}+c_{2}=\frac{K}{2} \leq \frac{\lambda}{2} .
\end{aligned}
$$

Dès lors on voit que pour tout $x \in \bigcup_{\theta \in \Delta} Q_{\theta}$ on a $\left|\pi^{I}\left(F \mathbb{1}_{V}, x\right)\right| \geq \lambda / 2$ et $A_{\alpha}^{I}\left(F \mathbb{1}_{V}, x\right) \leq 1$.

Et donc, pour tout $\mu \in \mathbb{R}^{+}$,

$$
\begin{aligned}
|E| & \leq c \sum_{\theta \in \Delta}\left|Q_{\theta}\right| \\
& \leq c \sum_{\theta \in \Delta} \exp \left(-\mu \frac{\lambda}{2}+c_{4} \mu^{2}\right) \int_{Q_{\theta}} d x \exp \left(\mu\left|\pi^{I}\left(F \mathbb{1}_{V}, x\right)\right|-c_{4} \mu^{2} A_{\alpha}^{I}\left(F \mathbb{1}_{V}, x\right)^{2}\right)
\end{aligned}
$$


$\leq c c_{5} \exp \left(-\mu \frac{\lambda}{2}+c_{4} \mu^{2}\right)|Q| \quad$ d'après la proposition 2.5

$\leq c c_{5} \exp \left(-\frac{\lambda^{2}}{16 c_{4}}\right)|Q| \quad$ si on prend $\mu=\frac{\lambda}{4 c_{4}}$.

Démonstration du théorème 2.7. Posons $a=2 c_{2}+c_{1}$ (cf. lemme 2.1).

Quitte à prendre $c_{9}>e^{36 c_{8} a^{2}}$, on peut se contenter de montrer le résultat pour $\gamma>2(a \delta+1)$ (et toujours $\gamma>3)$. Notons :

$$
\begin{aligned}
E_{1} & =\left\{\theta \in \mathbb{R}^{n} ; A_{\alpha}(F, \theta) \leq \delta \lambda\right\}, \\
E_{2} & =\left\{\theta \in \mathbb{R}^{n} ; \bar{\pi}(F, \theta)>\gamma \lambda \text { et } A_{\alpha / 3}(F, \theta) \leq \delta \lambda\right\}, \\
W_{1} & =\bigcup_{\theta \in E_{1}} \Gamma_{\alpha / 3}(\theta), \quad F_{1}=F \mathbb{1}_{W_{1}}, \\
E_{3} & =\left\{\theta \in \mathbb{R} ; \bar{\pi}\left(F_{1}, \theta\right)>\gamma \lambda\right\} \supset E_{2} .
\end{aligned}
$$

Remarquons que par construction de $W_{1}$ et le choix des ouvertures des cônes, pour tout $x \in \mathbb{R}^{n}$ il existe $\theta \in E_{1}$ tel que $\Gamma_{\alpha / 3}(x) \cap W_{1} \subset \Gamma_{\alpha}(\theta)$ et donc

$$
\left\|A_{\alpha / 3}\left(F_{1}\right)\right\|_{\infty} \leq \delta \lambda
$$

À chaque $\theta \in E_{3}$, on associe comme dans le lemme 2.9 un domaine lipschitzien $V_{\theta}$ de $\Gamma(\theta)$ tel que $\left|\pi\left(F_{1} \mathbb{1}_{V_{\theta}}, \theta\right)\right|=\bar{\pi}\left(F_{1} \mathbb{1}_{V_{\theta}}, \theta\right)=\left(1+c_{2} \delta\right) \lambda$. Cette fois on prend pour $Q_{\theta}$ le cube dyadique contenant $\theta$ et de longueur $\sup \left\{2^{k} ; k \in \mathbb{Z}, \sqrt{n} 2^{k}<y_{V_{\theta}}\right\}$.

D'après (1) et les propriétés de régularité de $\pi$ (lemme 2.1) on voit donc que

$$
\begin{aligned}
\left|\bar{\pi}\left(F_{1} \mathbb{1}_{V_{\theta}}, x\right)-\left(1+c_{2} r \delta\right) \lambda\right| & =\left|\bar{\pi}\left(F_{1} \mathbb{1}_{V_{\theta}}, x\right)-\bar{\pi}\left(F_{1} \mathbb{1}_{V_{\theta}}, \theta\right)\right| \\
& \leq c_{2} \frac{|\theta-x|}{y_{V_{\theta}}} A\left(F_{1} \mathbb{1}_{V_{\theta}}, \theta\right) \leq c_{2} \delta \lambda, \\
\bar{\pi}^{\left[3 \ell\left(Q_{\theta}\right),+\infty[\right.}\left(F_{1} \mathbb{1}_{V_{\theta}^{c}}, x\right) & \leq c_{1} A^{\left[3 \ell\left(Q_{\theta}\right),+\infty[\right.}\left(F_{1}, x\right) \leq c_{1} \delta \lambda
\end{aligned}
$$

(car on vérifie aisément que pour $x \in Q_{\theta}, y_{V_{\theta}}(x) \leq \frac{3}{2} y_{V_{\theta}} \leq 3 \ell\left(Q_{\theta}\right)$ ). D'où

$$
\begin{aligned}
& \bar{\pi}^{\left[3 \ell\left(Q_{\theta}\right),+\infty[\right.}\left(F_{1}, x\right) \leq \bar{\pi}^{\left[3 \ell\left(Q_{\theta}\right),+\infty[\right.}\left(F_{1} \mathbb{1}_{V_{\theta}^{\mathrm{c}}}, x\right)+\bar{\pi}^{\left[3 \ell\left(Q_{\theta}\right),+\infty[\right.}\left(F_{1} \mathbb{1}_{V_{\theta}}, x\right) \\
& \leq\left[\left(c_{1}+2 c_{2}\right) \delta+1\right] \lambda=(a \delta+1) \lambda .
\end{aligned}
$$

Puisque $\gamma>2(a \delta+1)$, on en conclut que

$$
\begin{aligned}
E_{3} \cap Q_{\theta} & =\left\{x \in Q_{\theta} ; \bar{\pi}\left(F_{1}, x\right)>\gamma \lambda\right\} \\
& \subset\left\{x \in Q_{\theta} ; \bar{\pi}^{] 0,3 \ell\left(Q_{\theta}\right)\right]}\left(F_{1}, x\right)>\gamma \lambda / 2\right\} .
\end{aligned}
$$

Comme les $Q_{\theta}, \theta \in E_{3}$, forment un recouvrement dyadique de $E_{3}$, on en extrait un sous-recouvrement $\Delta$ de $E_{3}$ de cubes essentiellement disjoints. Ainsi 


$$
\begin{aligned}
\left|E_{2}\right| & \leq\left|E_{3}\right| \leq \sum_{\theta \in \Delta}\left|E_{3} \cap Q_{\theta}\right| \\
& \leq \sum_{\theta \in \Delta}\left|\left\{x \in Q_{\theta} ; \bar{\pi}^{\left.00,3 \ell\left(Q_{\theta}\right)\right]}\left(F_{1}, x\right)>\gamma \lambda / 2\right\}\right| \quad \text { d'après (2) } \\
& \leq \sum_{\theta \in \Delta} c_{7} \exp \left(-c_{6}\left(\frac{\gamma}{2 \delta}\right)^{2}\right)\left|Q_{\theta}\right| \\
& \leq c_{7} \exp \left(-\frac{c_{6}}{4}\left(\frac{\gamma}{\delta}\right)^{2}\right)\left|\left\{x \in \mathbb{R}^{n} ; \bar{\pi}(F, x)>\lambda\right\}\right|
\end{aligned}
$$

d'après le théorème 2.6 , et grâce à (1).

Démonstration du corollaire 2.8. (a) : C'est une conséquence, selon des méthodes classiques, de l'inégalité aux bons- $\lambda$ du théorème 2.7 (voir [14] par exemple) : en l'intégrant en $\lambda$ après avoir multiplié par $p \lambda^{p-1} d \lambda$, on obtient après quelques manipulations (on utilise notamment que $\left\|A_{\alpha}(F)\right\|_{p} \leq$ $\left.c_{\alpha}\|F\|_{T_{p}}\right)$ que

$$
\gamma^{-p} \int_{\mathbb{R}^{n}} d \theta \bar{\pi}(F, \theta)^{p} \leq \delta^{-p} \int_{\mathbb{R}^{n}} d \theta A_{\alpha}(F, \theta)^{p}+c_{9} e^{-c_{8}(\gamma / \delta)^{2}} \int_{\mathbb{R}^{n}} d \theta \bar{\pi}(F, \theta)^{p} .
$$

Pour $F \in T_{c}$, la finitude de ces intégrales permet d'obtenir l'estimation souhaitée. Pour conclure il suffit d'utiliser la densité de $T_{c}$ dans les $T_{p}$. L'estimation en $+\infty$ sur $c_{p}$ s'obtient par optimisations successives dans les choix de $\delta$ et $\gamma$ dans l'équation (3).

(b) : Ce résultat d'intégrabilité pour le rapport $\bar{\pi}(F) / A_{\alpha}(F)$ se déduit de l'inégalité aux bons- $\lambda$ par des méthodes dues à Murai et Uchiyama (cf. [16] et [9]).

(c) : C'est une conséquence directe de l'inégalité aux bons- $\lambda$ et de la relation suivante :

$$
\begin{aligned}
& \frac{1}{|Q|} \int_{Q} d \theta \exp \left(\bar{\pi}^{0, \beta \ell(Q)]}(F, \theta)-c A_{\alpha}^{] 0, \beta \ell(Q)]}(F, \theta)^{2}\right) \\
& =\frac{1}{|Q|} \int_{\mathbb{R}} d x \int_{\mathbb{R}} d y 2 c y e^{x-c y^{2}} \mid\left\{\theta \in Q ; \bar{\pi}^{] 0, \beta \ell(Q)]}(F, \theta)>x\right. \\
& \text { et } \left.A_{\alpha}^{] 0, \beta \ell(Q)]}(F, \theta) \leq y\right\} \mid .
\end{aligned}
$$

3. Extension au cas des espaces $H^{p}, 0<p<1$. Nous avons vu au paragraphe 2 que dans un certain sens, sous l'hypothèse $\left(\mathcal{H}_{1}\right)$ et pour $F \in T^{p}, 0<p<\infty$, les $\pi\left(F \mathbb{1}_{W}\right), W \in \mathcal{L}^{*}$, sont "uniformément" dans $L^{p}$. Le théorème 1.1 de Coifman, Meyer et Stein assure que pour $F \in T^{p}$, $0<p \leq 1$, et sous la condition supplémentaire $\left(\mathcal{H}_{p}\right)$, les $\pi\left(F \mathbb{1}_{W}\right)$ peuvent être vus comme des éléments de $H^{p}$. La question se pose alors de savoir si ces $\pi\left(F \mathbb{1}_{W}\right), W \in \mathcal{L}$, sont "uniformément" dans $H^{p}$. C'est ce que nous allons établir. 
Il convient pour cela de modifier notre fonction maximale. Considérons $\psi \in \mathbb{C}^{\infty}\left(\mathbb{R}^{n}, \mathbb{R}_{+}\right)$à support compact (dans la boule unité par exemple) et la fonction maximale associée, définie pour toute distribution $f$ sur $\mathbb{R}^{n}$ par

$$
N[f](\theta)=\sup _{(x, y) \in \Gamma(\theta)}\left|\psi_{y} * f(x)\right| .
$$

Et introduisons :

DÉfinition 3.1. Pour toute fonction tente $F \in T^{p}, 0<p<\infty$, et tout $\theta \in \mathbb{R}^{n}$, on pose

$$
M \pi(F, \theta)=\sup _{W \in \mathcal{L}} N\left[\pi\left(F \mathbb{1}_{W}\right)\right](\theta) .
$$

Remarquons que dans ce contexte (i.e. si la condition d'annulation $\left(\mathcal{H}_{p}\right)$ est vérifiée) $\sup _{W \in \mathcal{L}}$ et $\sup _{W \in \mathcal{L}^{*}}$ se confondent. En effet (d'après le théorème 1.1 de Coifman, Meyer et Stein),

$$
\left|\psi_{y} * \pi\left(F \mathbb{1}_{W^{[0, \varepsilon]}}, x\right)\right|^{p} \leq \frac{c}{y^{n}}\left\|N\left(\pi\left(F \mathbb{1}_{W^{[0, \varepsilon]}}\right)\right)\right\|_{p}^{p} \leq \frac{c}{y^{n}}\left\|F \mathbb{1}_{W^{[0, \varepsilon]}}\right\|_{T_{p}}^{p} \stackrel{\varepsilon \rightarrow 0}{\longrightarrow} 0 .
$$

Proposition 3.2. Si $0<p \leq 1$, et si $\phi$ vérifie la condition d'annulation $\left(\mathcal{H}_{p}\right)$ associée, alors il existe une constante $c_{p}$ ne dépendant que de $n, \phi, \psi$, et $p$ telle que $\|M \pi(F)\|_{p} \leq c_{p}\|F\|_{T^{p}}$. Il en va de même si $1<p<\infty$ et si $\phi$ vérifie la condition d'annulation $\left(\mathcal{H}_{1}\right)$.

Évidemment cela est encore vrai pour tout choix raisonnable de fonction maximale $N$, et notamment pour la grande fonction maximale.

Démonstration. Le cas $p>1$ se déduit directement de l'inégalité maximale du paragraphe précédent (cf. corollaire 2.8(a)) et du fait que $M \pi(F) \leq$ $N(\bar{\pi}(F))$.

Dans le cas $0<p \leq 1$, il suffit de vérifier le résultat sur les atomes de $T^{p}$ (cf. [18, p. 106] par exemple). Soit donc $B$ une boule de $\mathbb{R}^{n}$ (de centre $c_{B}$ et de rayon $r_{B}$ ) et $a \in T^{2}$ une fonction tente à support dans la tente au-dessus de $B, T(B)=\left\{(x, y) \in \mathbb{R}_{+}^{n+1} ;\left|x-c_{B}\right|+y<r_{B}\right\}$, et telle que

$$
\|a\|_{T^{2}}^{2}=\int_{T(B)} y|a(x, y)|^{2} d x d y<|B|^{1-2 / p} .
$$

Sur $2 B$, la boule de même centre que $B$ et de rayon double, l'estimation souhaitée s'obtient grâce à l'inégalité de Hölder, à l'inégalité maximale $\|N(f)\|_{p} \leq c_{p}\|f\|_{p}$, et à l'inégalité maximale du paragraphe 2 (cf. corollaire $2.8(\mathrm{a}))$ :

$$
\begin{aligned}
\int_{2 B} M \pi(a, \theta)^{p} d \theta & \leq \int_{2 B} N[\bar{\pi}(a)](\theta)^{p} d \theta \leq\left(\int_{2 B} N[\bar{\pi}(a)](\theta)^{2} d \theta\right)^{p / 2}|2 B|^{1-p / 2} \\
& \leq c_{n}\|a\|_{T^{2}}^{p}|2 B|^{1-p / 2} \leq c_{n, p} .
\end{aligned}
$$


Hors de $2 B$, on utilise les conditions d'annulation $\left(\mathcal{H}_{p}\right)$. Soit $q_{x, y}(s)$ le polynôme de degré $d=\lfloor n / p-n\rfloor$ issu du développement de Taylor de la fonction $s \mapsto \psi_{y}(x-s)$ au point $c_{B}$. On a l'estimation habituelle

$$
\left|\psi_{y}(x-s)-q_{x, y}(s)\right| \leq c \frac{\left|s-c_{B}\right|^{d+1}}{y^{n+d+1}} .
$$

On voit donc que, pour tout $W \in \mathcal{L}$,

$$
\begin{aligned}
\left|\psi_{y} * \pi\left(a \mathbb{1}_{W}\right)(x)\right| & \leq \int_{B}\left|\pi\left(a \mathbb{1}_{W}, s\right)\right|\left|\psi_{y}(x-s)-q_{x, y}(s)\right| d s \\
& \leq c\left\|\pi\left(a \mathbb{1}_{W}\right)\right\|_{2} \frac{r_{B}^{n / 2+d+1}}{y^{n+d+1}} .
\end{aligned}
$$

D'où

$$
M \pi(a, x) \leq c_{n}\|a\|_{T^{2}} \frac{r_{B}^{n / 2+d+1}}{\left|x-c_{B}\right|^{n+d+1}} \quad \text { si } x \notin 2 B .
$$

Puisque $p(n+d+1)>n$, on en déduit que $\int_{\mathbb{R}^{n}} M \pi(a, x)^{p} d x \leq c_{n, p}$.

\section{Application à la définition ponctuelle de $\pi$}

Proposition 4.1. Supposons la condition d'annulation $\left(\mathcal{H}_{1}\right)$ vérifiée. Soit $F \in \bigcup_{0<p<\infty} T^{p}$. Pour presque tout $\theta \in \mathbb{R}^{n}$, la limite ci-dessous existe et permet donc de définir l'application $\pi_{f}$ :

$$
\pi_{f}: T^{p} \rightarrow L^{p}, \quad F \mapsto \pi_{f}(F, \theta):=\lim _{\substack{V \rightarrow \mathbb{R}_{+}^{n+1} \\ V \in \mathcal{L}^{*}}} \pi\left(F \mathbb{1}_{V}, \theta\right) .
$$

L'opérateur $\pi_{f}$ est alors une extension continue de $\pi$ de $T^{p}$ sur $L^{p}$.

- Si $p \geq 1$, la fonction $\pi_{f}(F)$ ainsi définie est un représentant dans $L^{p}$ de la fonction $\pi(F)$ définie (par dualité) par Coifman, Meyer et Stein dans [8] (voir aussi le théorème 1.1).

- Si $0<p<1$, et si la condition d'annulation supplémentaire $\left(\mathcal{H}_{p}\right)$ est vérifiée, alors les $\pi(F)$ tels qu'ils sont définis par Coifman, Meyer et Stein sont des distributions appartenant à $H^{p}$. Nos $\pi_{f}(F)$ sont alors la "partie fonction" de ces distributions, c'est-à-dire des représentants dans $L^{p}$ des limites non-tangentielles des extensions harmoniques des $\pi(F)$.

Remarquons que dans le cas où $0<p \leq n /(n+1)$ et où seule la condition d'annulation $\left(\mathcal{H}_{1}\right)$ est vérifiée, les $\pi_{f}(F)$ sont bien définis alors que les $\pi(F)$ ne le sont pas.

Démonstration de la proposition 4.1. Un schéma de preuve classique ([17, pp. 8-9]) montre à partir de l'inégalité maximale de la section 2 (cf. corollaire 2.8(a)) que pour presque tout $\theta \in \mathbb{R}^{n}$, la limite (4) existe et est uniforme sur $\mathcal{L}^{*}$.

Rappelons la démarche dans notre contexte. 
Lemme 4.2. Pour tout $F \in \bigcup_{0<p<\infty} T^{p}$ et presque tout $\theta \in \mathbb{R}^{n}$ on a

$$
\Lambda(F, \theta):=\limsup _{t \rightarrow 0} \bar{\pi}^{0, t]}(F, \theta)=0 .
$$

Démonstration du lemme 4.2. Constatons tout d'abord que $\Lambda(F, \theta)=0$ en tout $\theta \in \mathbb{R}^{n}$ dès que $F \in T_{c}$.

Notons aussi que pour $t>0$ et $F \in T^{p}$ on a $\bar{\pi}^{0, t]}(F, \theta) \leq 2 \bar{\pi}(F, \theta)$. Dès lors pour tout $\varepsilon>0$, on peut trouver $G \in T_{c}$ tel que $\|F-G\|_{T^{p}} \leq \varepsilon$ et ainsi $\Lambda(F, \theta) \leq \Lambda(F-G, \theta)+\Lambda(G, \theta)=\Lambda(F-G, \theta) \leq 2 \bar{\pi}(F-G, \theta)$. D'où $\|\Lambda(F)\|_{p} \leq 2\|\bar{\pi}(F-G)\|_{p} \leq 2 c_{p}\|F-G\|_{T^{p}} \leq 2 c_{p} \varepsilon$ d'après l'inégalité maximale (cf. corollaire 2.8(a)). Et donc $\|\Lambda(F)\|_{p}=0$.

Pour presque tout $\theta \in \mathbb{R}^{n}, V \mapsto \pi\left(F \mathbb{1}_{V}, \theta\right)$ se prolonge donc par continuité à $\mathcal{L}$. La limite $(4)$ est donc bien définie.

Montrons maintenant que, sous les hypothèses de la proposition, elle cö̈ncide avec les limites non-tangentielles. On peut se contenter de montrer le résultat pour des extensions associées à un noyau $\Psi$ comme celui que nous avons utilisé dans la section 3 et vérifiant de plus $\int \Psi=1$. Notons alors

$$
\pi_{\mathrm{NT}}(F, \theta)=\lim _{\substack{z \in \Gamma(\theta) \\ z \rightarrow \theta}} \Psi_{y} * \pi(F)(x) .
$$

Considérons les ensembles $E_{n}=\left\{\theta \in \mathbb{R}^{n} ; \bar{\pi}(F, \theta) \leq n, A_{3}(F, \theta) \leq n\right\}$, $W_{n}=\bigcup_{\theta \in E_{n}} \Gamma(\theta)$ et $\Omega_{n}=W_{n}^{\mathrm{c}}$. Par construction $\left\|A\left(F \mathbb{1}_{W_{n}}\right)\right\|_{\infty} \leq n$ et (c'est une simple conséquence du lemme $2.1(\mathrm{~b}))\left\|\bar{\pi}\left(F \mathbb{1}_{W_{n}}\right)\right\|_{\infty} \leq\left(1+c_{2}\right) n$. On est ainsi ramené à des fonctions intégrables, pour lesquelles il est clair que $\pi\left(F \mathbb{1}_{W_{n}}\right)=\pi_{f}\left(F \mathbb{1}_{W_{n}}\right)$. On a donc

$$
\left|\pi_{\mathrm{NT}}(F, \theta)-\pi_{f}(F, \theta)\right| \leq M \pi\left(F \mathbb{1}_{\Omega_{n}}, \theta\right)+\bar{\pi}\left(F \mathbb{1}_{\Omega_{n}}, \theta\right) .
$$

Les inégalités maximales du corollaire 2.8(a) et de la proposition 3.2 impliquent que

$$
\left\|\pi_{\mathrm{NT}}(F)-\pi_{f}(F)\right\|_{p} \leq 2 c_{p}\left\|F \mathbb{1}_{\Omega_{n}}\right\|_{T_{p}},
$$

ce qui tend vers zéro quand $n$ tend vers l'infini (puisque $\bigcap_{n \in \mathbb{N}} \Omega_{n}=\emptyset$ ).

\section{Références}

[1] R. Bañuelos, I. Klemes, and C. N. Moore, An analogue for harmonic functions of Kolmogorov's law of the iterated logarithm, Duke Math. J. 57 (1988), 37-68.

[2] R. Bañuelos and C. N. Moore, Laws of the iterated logarithm, sharp good- $\lambda$ inequalities and $L^{p}$-estimates for caloric and harmonic functions, Indiana Univ. Math. J. 38 (1989), 315-344.

[3] - - - Sharp estimates for nontangential maximal function and the Lusin area function in Lipschitz domains, Trans. Amer. Math. Soc. 312 (1989), 641-662.

[4] -, - Distribution function inequalities for the density of the area integral, Ann. Inst. Fourier (Grenoble) 41 (1991), 137-171. 
[5] M. T. Barlow and M. Yor, Semi-martingale inequalities via the Garsia-RodemichRumsey lemma, and applications to local times, J. Funct. Anal. 49 (1982), 198-229.

[6] S.-Y. A. Chang, J. M. Wilson and T. H. Wolff, Some weighted norm inequalities concerning the Schrödinger operators, Comment. Math. Helv. 60 (1985), 217-246.

[7] L. Chevalier, Une "formule de Tanaka" en analyse harmonique et quelques applications, Adv. Math. 138 (1998), 182-210.

[8] R. R. Coifman, Y. Meyer and E. M. Stein, Some new function spaces and their applications to harmonic analysis, J. Funct. Anal. 62 (1985), 304-335.

[9] R. Fefferman, R. Gundy, M. Silverstein and E. M. Stein, Inequalities for ratios of functionals of harmonic functions, Proc. Nat. Acad. Sci. U.S.A. 79 (1982), 79587960.

[10] R. F. Gundy, Some Topics in Probability and Analysis, CBMS Regional Conf. Ser. in Math. 70, Amer. Math. Soc., 1989.

[11] R. F. Gundy and M. L. Silverstein, The density of the area integral in $\mathbb{R}_{+}^{n+1}$, Ann. Inst. Fourier (Grenoble) 35 (1985), 215-229.

[12] E. Labeye-Voisin, Espaces de tentes, principe de domination et application à l'étude de la densité de l'intégrale d'aire, thèse de doctorat de l'Université Joseph Fourier, Grenoble I, 1999.

[13] —, Régularité de la densité de l'intégrale d'aire, Potential Anal. (2002), à paraître.

[14] E. Lenglart, D. Lepingle et M. Pratelli, Présentation unifiée de certaines inégalités de la théorie des martingales, dans : Séminaire de probabilités XIV, 1978/79, Lecture Notes in Math. 784, Springer, 1980, 26-48.

[15] C. N. Moore, Some inequalities for the density of the area integral, dans : B. Dahlberg et al. (eds.), Partial Differential Equations with Minimal Smoothness and Applications (Chicago, IL, 1990), IMA Vol. Math. Appl. 42, Springer, 1992, 189-198.

[16] T. Murai and A. Uchiyama, Good $\lambda$ inequalities for the area integral and the nontangential maximal function, Studia Math. 83 (1985), 251-262.

[17] E. M. Stein, Singular Integrals and Differentiability Properties of Functions, Princeton Univ. Press, 1970.

[18] —, Harmonic Analysis: Real-Variable Methods, Orthogonality, and Oscillatory Integrals, Princeton Math. Ser. 43, Princeton Univ. Press, 1993.

U.M.P.A., E.N.S. de Lyon

46 Allée d'Italie

69364 Lyon, France

E-mail: elabeye@voila.fr

Received June 27, 2000

Revised version June 17, 2002 